\title{
Editorial
}

\section{Advancing collaborative practice and preventive culture in work and community life}

The importance of advancing proactive health-related practice and preventive culture is recognized in increasingly diversifying work and community life. The new journal "Environmental and Occupational Health Practice" is launched as a unique forum for promoting the exchange of reports and studies on onsite practices related to health and environment in work and community life. The knowledge gained through this forum is expected to provide useful hints and clues for developing action-oriented strategies and guidelines in environmental and occupational health actions.

The emphasis of environmental and occupational health activities today is commonly placed on proactive risk assessment and control. This requires effective risk management processes dealing with multifaceted risks in real life as well as well-informed support services. Awareness is thus growing that good practices in upgrading occupational health and safety and environmental quality in varied work and life situations can offer broadly applicable guidance for risk management and support services effective and valid in diverse situations. The outcomes gained through new initiatives and adjustments for work and life practices can likewise be useful sources of key information about improving existing practices. The studies reporting immature results may also help people and practitioners understand better ways of building meaningful practices.

Recent experiences particularly in occupational health fields point to the need to manage multifaceted health and safety risks in a comprehensive manner by locally adjusted procedures aimed at sustained preventive culture. These experiences confirm the merit of nurturing collaborative practice among people concerned by means of applying practical tools that can involve local people and lead to effective preventive actions. Participatory action-oriented approaches developing in various sectors for preventing multifaceted risks at work and daily life also demonstrate the advantage of locally adjusted procedures and tools for facilitating primary prevention particularly in small-scale workplaces. It is hoped that this new journal will promote the discussion and development of both these aspects of locally effective proactive procedures and action-oriented tools that have real impact in the local context.

The start of this new journal is also expected to elucidate the practical ways of securing effective teams of occupational health services and environmental activities. It is important to focus on the support activities that can promote primary prevention of variably significant multiple risks and on the active roles of managers, workers and practitioners in securing effective participatory steps. In this context, support services through action-oriented tools and training is essential rather than through scaling methods and analytical procedures alone.

Attention is also drawn to the link between recent developments in international standards in risk management supported by people-centered systems and varied health-related practices in different local situations. The developments in risk control process norms clearly reflect the spreading need to prevent systems-related accidents and disorders, and hence require effective collaboration practices adapted to each local situation. The international trends in advocating people-centered methods seem particularly relevant. In supporting these trends, the changing roles of occupational health and safety services and the varied participatory forms of promoting preventive culture in locally adjusted manners are likewise noteworthy. The different aspects of related local practices as well as their effectiveness in meeting the norms and trends with concrete outcomes may require in-depth studies. In view of this link, field studies on local practices may lead to improving conditions of work and environment in underserved sectors, small-scale and informal workplaces and newly emerging work life situations involving migrant workers, aged workers and people with different support needs. The networking of good-practice approaches relying on effective people-centered systems can be advanced through these local-context studies.

The launch of this journal surely gives new impetus towards exploring the spread of good practices and a culture of sustainable prevention in the workplace and in the community. We are confident that the journal will make significant contribution to the quality of research and practice aimed at our common goals. We are pleased to join many practitioners and researchers in environmental and occupational health fields in supporting the journal. 